\title{
Isolation und Anreicherung von Krankheitserregern mit Hilfe eines oberflächenmodifizierten Aluminium-Chips für Raman-spektroskopische Anwendungen
}

\author{
Susanne Pahlow ${ }^{1,2}$, Sandra Kloß ${ }^{1}$, Verena Blättel ${ }^{3}, K_{\text {Konstantin Kirsch }}^{1}$, Uwe Hübner ${ }^{1}$, Dana Cialla ${ }^{1,2}$, \\ Petra Rösch ${ }^{1}$, Karina Weber ${ }^{1,2}$, Jürgen Popp ${ }^{1,2}$ \\ ${ }^{1}$ Institut für Photonische Technologien Jena, Albert-Einstein-Str. 9, D-07745 Jena, \\ Juergen.Popp@ipht-jena.de \\ ${ }^{2}$ Institut für Physikalische Chemie, Friedrich-Schiller-Universität Jena und Abbe-Center of Photonics, \\ Helmholtzweg 4, D-07743 Jena \\ ${ }^{3}$ R-Biopharm AG, An der neuen Bergstraße 17, D-64297 Darmstadt
}

\begin{abstract}
Zusammenfassung
Wir haben einen Raman-kompatiblen Chip zur Isolation von Mikroorganismen aus komplexen Medien entwickelt. Die Isolation der Bakterien wird unter Verwendung von Antikörpern als Fängermoleküle erreicht. Aufgrund der hochspezifischen Wechselwirkung mit den Zielmolekülen ist dies ein sehr vielversprechender Ansatz um Bakterien auch aus komplexen Matrices wie Körperflüssigkeiten zu isolieren. Die Fängermoleküle wurden so gewählt, dass auch die Untersuchung von Proben, die bisher noch nicht identifizierte Bakterien enthalten, ermöglicht wird. Die verwendeten Antikörper binden an gemeinsame Zellwandstrukturen Gram-positiver bzw. Gram-negativer Organismen und erlauben es daher eine ganze Bandbreite an Bakterien mit nur zwei verschiedenen Antikörpern zu isolieren. Die Anwendbarkeit unseres Chips wurde für verschiedene Gram-positive und Gramnegative Keime nachgewiesen.
\end{abstract}

Keywords: Bakterienidentifikation, komplexe Medien, Raman-Mikrospektroskopie und Chip-basierte Isolierung.

\section{Einführung}

In vielen verschiedenen analytischen Anwendungsfeldern ist sowohl eine sehr schnelle als auch zuverlässige Erkennung von Krankheitserregern erforderlich. [1,2] In der medizinischen Diagnostik beispielsweise kann eine schnelle Identifizierung von Sepsiserregern die Überlebenschancen für den Patienten dramatisch verbessern. [3] Je früher der behandelnde Arzt die für die Infektion verantwortlichen Erreger ermitteln kann, desto schneller kann die entsprechende Therapie mit geeigneten Antibiotika eingeleitet werden. Derzeit erfordert die empfohlene Methode zur Diagnose von Sepsis noch Blutkulturen. [4] Dieser Ansatz ist nicht nur zeitaufwändig, sondern es besteht auch das Risiko falschnegativer Ergebnisse, da die Erreger nicht unbedingt im Blut des Patienten vorhanden sein müssen.

Im Vergleich zu auf PCR (polymerase chain reaction) basierenden Methoden, die ein sensitives und spezifisches Werkzeug zur Identifizierung von Krankheitserregern sind, könnte die Raman-Mikroskopie ein noch attraktiverer Ansatz für die labelfreie Detektion von Bakterien auf Spezies-Ebene sein. Die Raman-Spektroskopie ermöglicht eine schnelle und zuverlässige Detektion von Bakterien aufgrund des für die einzelnen Spezies charakteristischen Raman-Spektrums. [5] Um die intrinsisch schwachen Raman-Signale zu verstärken können verschiedene Techniken zum Einsatz kommen. Dazu zählen die oberflächenverstärkte Raman-Spektroskopie (SERS - surface enhance Raman scattering) [6] und die Ausnutzung des Resonanz-RamanEffekts. [7] Bevor jedoch Raman-Messungen an einzelnen Zellen durchgeführt werden können, müssen die Zellen von der sie umgebenden Matrix isoliert werden. Auf diese Weise ist es möglich die Spektren ohne den störenden Einfluss anderer Substanzen aufzunehmen. Für viele Einsatzgebiete ist dies generell schwierig, da die Zusammensetzung der Proben oft komplex ist. Aus diesem Grund haben wir uns für Antikörper als Fänger-Moleküle entschieden. $\mathrm{Da}$ die Antikörper-AntigenWechselwirkung eine sehr hohe Spezifität aufweist, sind Antikörper ideal zum Isolieren 
von Zellen oder Molekülen auch aus einer komplexen Umgebung. Allerdings kann die hohe Spezifität von Antikörpern in einem klassischen Immunosensor ein Nachteil sein, weil mehrere Antikörper benötigt werden, wenn die Probe auf mehrere Bakterienspezies hin gleichzeitig untersucht werden soll. Zudem kann es eine Herausforderung sein, pathogenspezifische Antikörper zu finden, da die Antikörper oft bedingt durch gemeinsame Strukturen in der Zelloberfläche wie Proteine oder Kohlenhydrate nur auf Gattungsebene spezifisch sind. In unserem Ansatz nutzen wir diese Tatsache zu unserem Vorteil aus, indem wir gezielt Antikörper gegen gemeinsame Zellwandstrukturen von Gram-positiven und Gram-negativen Bakterien verwenden. Die ausgewählten Antikörper binden an Lipopolysaccharide, welche sich auf der Zelloberfläche Gram-negativer Bakterien befinden und Lipoteichonsäuren, die in der Zellwand Gram-positiver Bakterien vorhanden sind. Mit diesen zwei Antikörpern kann damit ein sehr breites Spektrum von Bakterien isoliert werden. Vor einiger Zeit berichteten Maeng et al. von einer Methode zum fluoreszenzbasierten Nachweis von Gram-positiven und Gram-negativen Bakterien durch die Verwendung von RNA-Aptameren, die sich auch auf die Anbindung an die Zellwandkomponenten Lipopolysaccharide und Teichonsäuren stützt. [8] Im Gegensatz zu Antikörpern sind RNA-Aptamere jedoch verhältnismäßig instabile Fängermoleküle. Der wesentliche Vorteil unseres Ansatzes ist die labelfreie Identifizierung einzelner Bakterienzellen über Raman-Messungen. Daher können Proben mit beliebigen Zusammensetzungen von Bakterien untersucht werden. Auch eine Probe, die beispielsweise zwei verschiedene Spezies enthält, stellt keinerlei Probleme dar, da jedes aufgenommene Einzelzellspektrum einzeln mit einer Datenbank abgeglichen und entsprechend zugeordnet wird. Hierbei nutzen wir die Tatsache aus, dass jede Spezies ein charakteristisches Spektrum aufweist, auf dessen Grundlage eine einzelne Bakterienzelle eindeutig unterschieden und zugeordnet werden kann, wodurch zusätzliche fluoreszenzmarkierte Aptamere oder Antikörper unnötig werden. Die Voraussetzung für die Identifizierung einzelner Bakterienzellen in Proben ist eine chemometrische Analyse unter Verwendung einer Datenbank, die eine ausreichend große Menge von Spektren der für die entsprechende Anwendung relevanten Bakterienarten enthält. Die Raman-Messungen der isolierten Mikroorganismen können direkt auf dem Chip ausgeführt werden, da das für die
Beschichtung der Chipoberfläche verwendete Aluminium, selbst nicht Raman-aktiv ist. Durch den Einsatz von Aluminium können störende Hintergrundsignale vermieden werden, die sonst möglicherweise zu einer Fehlklassifikation der Bakterien führen würden.

Das Hauptziel dieser Arbeit ist es, eine einfache und schnelle Methode zur Chip-basierten Detektion von Mikroorganismen bereit zu stellen, um eine Raman-spektroskopische Identifizierung zu ermöglichen. Isolierung und Anreicherung der Bakterien werden mit einem miniaturisierten Mikrofluidikaufbau ermöglicht. Die Raman-Messungen werden direkt auf der Chipoberfläche durchgeführt. Die wichtigsten Vorteile unseres Ansatzes sind die Vielzahl verschiedener Bakterienarten, die aus den Proben isoliert werden können und die schnelle Verfügbarkeit der Ergebnisse, welche durch die Anwendung der Raman-Mikrospektroskopie erhalten werden. Weil die Raman-Mikroskopie die Identifizierung von Bakterien auf der Ebene einzelner Zellen ermöglicht, ist sie zudem eine Methode mit sehr hoher Empfindlichkeit.

\section{Experimenteller Teil}

Herstellung der Raman-Chips. Die RamanSubstrate werden auf 4"-Wafer-Level unter Reinraumbedingungen in einer Abfolge von Photolithographie, Magnetron-Sputtern und LiftOff-Technik hergestellt. Das komplette Waferlayout beinhaltet 37 nummerierte Chips. Jeder Chip ist $12,7 \times 12,7 \mathrm{~mm}^{2}$ groß und jedes Chiplayout enthält acht metallische Flächen von jeweils 1,5 x 1,5 $\mathrm{mm}^{2}$. Die StandardPhotolithographie wird unter Verwendung eines Mask-Aligners und einer Chrommaske als Umkehrprozess (Image-reversal) durchgeführt. Der Umkehrprozess ermöglicht gut hinterschnittene Resistmasken für den späteren Lift-Off-Prozeß. Die vorgereinigten Wafer ([100]-Si mit $1 \mu \mathrm{m}$ thermischen Oxid) werden mit 1,8 $\mu \mathrm{m}$ dickem Photoresists AZ5214E belackt und mit einer Dosis von $20 \mathrm{~mJ} / \mathrm{cm}^{2}$ belichtet. Nach dem Bildumkehrprozess ( $120{ }^{\circ} \mathrm{C}$ Temperung und Flutbelichtung mit $120 \mathrm{~mJ} / \mathrm{cm}^{2}$ ) und der Entwicklung in MIF 726 Entwickler (40 s) wird der Wafer mittels Magnetron-Sputtern mit einer $210 \mathrm{~nm}$ dicken Aluminium-Schicht beschichtet. Abschließend werden die metallischen Flächen durch einen Lift-Off-Prozess in einem Lösungsmittelbad herauspräpariert (Aceton-Bad, gefolgt von einer Isopropanol-Spülung).

Silanisierung. Vor der Silanisierung wurden die Chips mit Aceton, Ethanol und destilliertem Wasser gereinigt. Dazu wurden die Chips für jeweils $10 \mathrm{~min}$ im entsprechenden Lösungsmittel im Ultraschallbad inkubiert. Für 
die Silanisierung wurde eine Lösung von Ethanol mit $1 \mathrm{M}$ Essigsäure auf $\mathrm{pH} \quad 5,5$ eingestellt. Anschließend wurden $2 \%$ (v/v) (3-Glycidyloxypropyl)trimethoxysilan (sigma aldrich) zugefügt. Um die Entstehung reaktiver Silanolgruppen durch Hydrolyse zu ermöglichen, wurde die Lösung für 5 min mit Hilfe eines Magnetrührers durchmischt bevor die zu silanisierenden Substrate hinzugefügt wurden. Nach 30 min wurden die Substrate aus der Lösung entfernt und dreimal mit Ethanol gewaschen. Schließlich wurden die Chips bei $110^{\circ} \mathrm{C}$ für 20 min im Ofen ausgehärtet.

Modifizierung mit Antikörpern. Die Antikörper gegen Lipopolysaccharide (PAB14001) und Lipoteichon Säuren (MAB4715) wurden von Abnova (Taipei, Taiwan) bezogen. Vor dem Auftragen der Antikörper auf die Chips wurden diese auf eine Konzentration von $100 \mu \mathrm{g} / \mathrm{ml}$ mit 5x PBS (phosphate buffered saline) verdünnt. Das verwendete Volumen je Spot betrug $500 \mathrm{nl}$. Zunächst wurde für $3 \mathrm{~h}$ bei Raumtemperatur und anschließend über Nacht bei $4{ }^{\circ} \mathrm{C}$ in einer Feuchtkammer inkubiert. Die Chips wurden dann dreimal mit 1x PBS mit 0,1\% Tween 20 gewaschen und schließlich mit destilliertem Wasser abgespült.

Inkubation mit Bakterien. Kulturen von Bacillus subtilis DSM 6399, Escherichia coli DSM 423, Pseudomonas stutzeri DSM 5190, Pseudomonas putida DSM 291, Staphylococcus epidermidis RP62A, Staphylococcus cohnii DSM 20261, Enterococcus faecalis, Enterococcus faecium, Klebsiella pneumonaie, Pseudomonas aerusginosa, Staphylococcus aureus MRSA wurden für diese Studie verwendet. Sämtliche pathogene Bakterien wurden vor den Experimenten mit einer 4 prozentigen (v/v) Formaldehyd-Lösung in $1 x$ PBS Puffer inaktiviert.

Raman Messungen. Die Messungen wurden mit einem Raman-Mikroskop-Aufbau (BioParticleExplorer, rapID particle systems $\mathrm{GmbH}$, Berlin, Deutschland) durchgeführt. Die Wellenlänge betrug $532 \mathrm{~nm}$ und es wurde ein Objektiv mit 100x Vergrößerung verwendet (für mehr Informationen, siehe [5]). Die Intergrationszeit pro Spektrum betrug $10 \mathrm{~s}$.

\section{Chemometrische Analyse. Die} Vorverarbeitung der Rohdaten wurde mit Hilfe der ' $R$ ' Software [9] mit selbst generierten Skripten [10] durchgeführt. Der erste Schritt bestand in der Entfernung des Hintergrunds basierend auf einer Methode, die sich des SNIP clipping-Algorithmus bedient. [11] Für die chemometrische Analyse wurden die Wellenzahl-Regionen von $440-1799 \mathrm{~cm}^{-1}$ und
2650-3101 $\mathrm{cm}^{-1}$ benutzt. Alle Spektren wurden Vektor-normalisiert. Durch die Anwendung einer Hauptkomponentenanalyse (principal component analysis - PCA) konnte die Dimensionalität des Datensatzes reduziert werden, so dass schließlich nur 13 erste Hauptkomponenten für die weitere Statistik verwendet wurden. Für die Klassifizierung der Einzelzellspektren wurde eine lineare Diskriminanzanalyse (linear discriminant analysis - LDA) angewendet. [12]

\section{Ergebnisse und Diskussion}

Biomodifizierung der Aluminium Substrate. Es ist bekannt, dass Antikörper leicht auf verschiedenen festen Trägern durch Adsorption immobilisiert werden können. [13] Obwohl dies eine sehr einfache Möglichkeit aufgrund der geringen experimentellen Anforderungen darstellt, erfordern die meisten Anwendungen eine stabilere Anbindung der Antikörper. Da der Raman-Chip zur Isolierung von Bakterien aus komplexen Matrices genutzt werden soll, müssen die immobilisierten Antikörper mehrere Waschschritte überstehen. Aus diesem Grund wurde eine kovalente Immobilisierungsmethode gewählt. Die Anbindung des Antikörpers erfolgt über das Organosilan (3Glycidyloxypropyltrimethoxysilan (GOPS)). Die GOPS modifizierten Aluminium-Substrate stellen reaktive Epoxidgruppen für die kovalente Bindung von Antikörper über ihre Amin-, Thiol- und Hydroxylgruppen zur Verfügung.

Isolation von Mikroorganismen mit dem Raman-Chip. Zunächst wurden die mit Antikörpern modifizierten Aluminiumsubstrate mit vergleichsweise hohen Konzentrationen von Bakterien (ca. $5 \times 10^{8}$ Zellen/ml) in PBS-Puffer inkubiert. In diesen Experimenten wurden nur sechs der acht Messfelder mit Antikörpern beschichtet. Damit sollte gezeigt werden, dass die Immobilisierung der Bakterien in der Tat durch die Antikörper hervorgerufen wird. Es wurde beobachtet, dass die Bakterien bevorzugt in den Antikörper-beschichteten Bereichen haften blieben. In Abb. 1 werden Bereiche der Oberfläche zweier Chips nach der Inkubation mit $B$. subtilis (Chip 1) oder E. coli (Chip 2) gezeigt. Dazu wurde ein Chip mit Antikörpern gegen Gram-positive Bakterien (1a, 1c) beschichtet, während der andere mit Antikörpern gegen Gram-negative Bakterien (1b, 1d) beschichtet wurde. Für beide Arten zeigen die mikroskopischen Bilder deutlich, dass die mit Antikörper beschichteten Felder (1c, 1d) bevorzugt werden. Es gibt jedoch auch einige unspezifisch adsorbierte Bakterien in den unbeschichteten Bereichen, wahrscheinlich weil 
die Chips mit einer hohen Zellkonzentration inkubiert wurden.

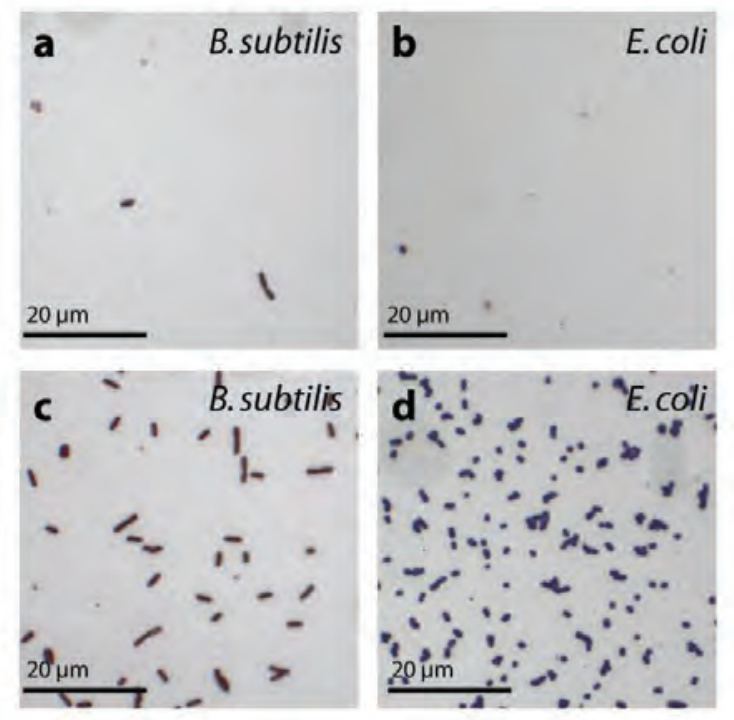

Abb. 1. Mikroskopische Aufnahmen zweier Chips welche mit Antikörpern für Gram-positive Bakterien (c, Chip 1) und mit Antikörpern für Gram-negative Bakterien (d, Chip 2) beschichtet wurden nach Inkubation mit E. coli oder B. subtilis. Zum Vergleich werden Bilder der unbeschichteten Bereiche auf dem gleichen Chip (a, b) gezeigt. Zur besseren Sichtbarkeit wurden die Bakterien mit Kristallviolett bzw. Karbolfuchsin gefärbt.

Raman Messungen der isolierten Bakterien. Die Funktion des Antikörper-modifizierten Raman-Chips wurde durch Isolierung verschiedener Bakterienarten aus PufferLösung untersucht. Alle untersuchten Arten konnten erfolgreich mit dem Chip isoliert werden, obwohl Unterschiede in der Affinität der Bakterien zu den Fänger-Antikörper festgestellt wurden. Raman-Messungen der isolierten Bakterien ergaben augenscheinlich typische Spektren (siehe Abb. 2). Alle Zellbestandteile wie Proteine, Lipide, DNA, RNA etc. tragen zum Spektrum bei. Beispielsweise zeigen alle untersuchten Spezies eine breite Bande, die bei $2900 \mathrm{~cm}^{-1}$ zentriert ist, und auf $\mathrm{CH}$-Streckschwingungen von $\mathrm{CH}_{2}$ - und $\mathrm{CH}_{3}$-Gruppen, die in Proteinen, Lipiden, Nukleinsäuren und Kohlenhydraten vorkommen, basiert.

Alle Spektren weisen zwei Bereiche mit großen Gemeinsamkeiten auf, die auf die Gegenwart von Proteinen in den Zellen zurückzuführen sind: Die Amid-I und Amid-III Banden bei 1670 und $1240 \mathrm{~cm}^{-1}$. Auch die sehr scharfe Bande bei $1000 \mathrm{~cm}^{-1}$, die Phenylalanin zugeschrieben werden kann, ist eine charakteristische Bande die in allen Bakterienspektren auftritt. Darüber hinaus wurden Messungen des spektralen Hintergrunds durchgeführt. Weder die gereinigte noch die silanisierte Aluminiumoberfläche zeigten signifikante Raman-Banden. Dasselbe gilt für die mit Antikörper modifizierten Messfelder, welche ebenfalls keine prominenten Raman-Banden aufweisen.

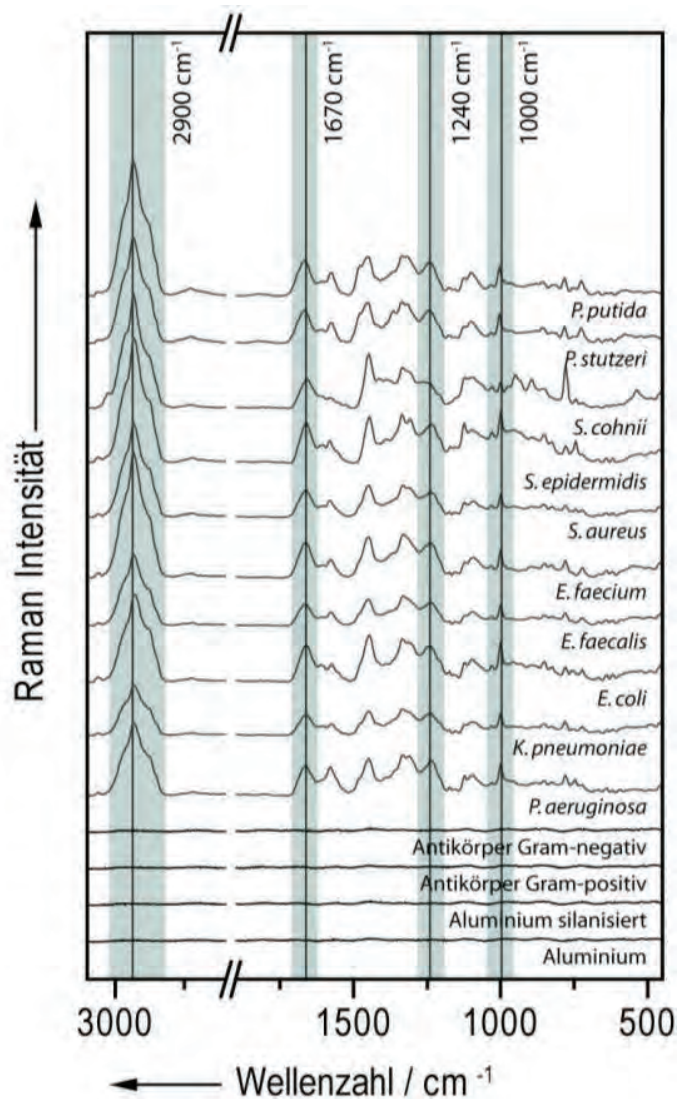

Abb 2. Hintergrund Spektren des Aluminiumsubstrats (a unbehandelt, b silanisiert, c mit anti-Gram-positive und $d$ anti-gramnegative Antikörper) und Hintergrund korrigierte Spektren (gemittelt aus mindestens 20 Einzelzell-Spektren) der untersuchten Spezies Aus Gründen der Übersichtlichkeit wurden die Spektren vertikal verschoben.

Für eine genauere Analyse wurden zusätzliche Spektren mit Nickelfolie als Referenz Substrat aufgenommen (siehe Abbildung 3). Nickelfolie ist ein etabliertes Substrat für RamanMessungen an Bakterien, da Nickel ebenfalls keine störenden Hintergrundsignale verursacht. [5] Zusätzlich zur Nickelfolie wurden die silanisierten Aluminiumsubstrate und die Antikörper-modifizierten Substrate ebenfalls mit $S$. aureus und $E$. faecalis versehen und zum Vergleich gemessen. Wie in Abbildung $3 \mathrm{zu}$ sehen ist, treten keine zusätzlichen Banden in den Spektren der silanisierten und mit dem Antikörper modifizierten Aluminiumsubstraten auf. Somit lässt sich festhalten, dass die dünnen, auf Silizium aufgetragenen AluminiumSchichten ein gut geeignetes Raman-Substrat darstellen. Selbst nach der Modifikation mit 
Antikörpern werden typische Bakterien Spektren erhalten.

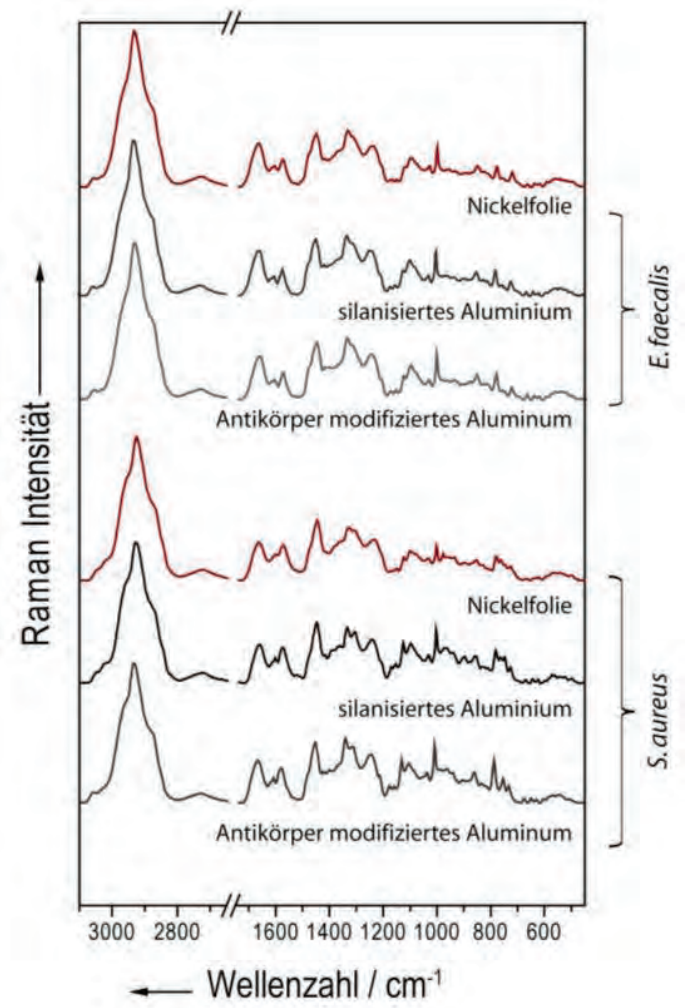

Abb 3. Vergleich der auf Nickelfolie, silanisiertem Aluminium und Antikörper-modifizierten Aluminium gemessen Raman Spektren.

Da geplant ist den Raman-Chip zur Isolierung und Identifizierung von Bakterien aus Proben wie Körperflüssigkeiten zu nutzen, muss sichergestellt werden, dass eine chemometrische Analyse der spektralen Daten möglich ist. Das Ziel der chemometrischen Analyse ist die Untersuchung der zum Teil sehr subtilen Unterschiede zwischen den Spektren verschiedener Bakterien, um sie zu unterscheiden und eindeutig zu identifizieren. Als einen ersten Schritt in Richtung dieses Ziels wurde ein kleiner Datensatz mit fünf verschiedenen pathogenen Bakterien zur Klassifizierung mittels Hauptkomponentenanalyse und der linearen Diskriminanzanalyse verwendet. Die Ergebnisse in Tabelle 1 zeigen, dass die Klassifizierung der gesammelten Daten prinzipiell funktioniert. Für eine praktische Anwendung unseres Verfahrens müssen allerdings noch weitere Experimente durchgeführt werden, um einen ausreichend große Datensatz zu erhalten, der für eine sichere Identifizierung eingesetzt werden kann.

Untersuchung von geringeren Zellkonzentrationen. In weiteren Experimenten wurden die Antikörper-modifizierten Chips mit abnehmenden Konzentrationen von B. subtilis und $S$. epidermidis in Pufferlösung inkubiert.
Nach gründlichem Waschen wurden die Substrate mit Hilfe des Raman-Setups untersucht. Bis zu Konzentrationen von $5 \times 10^{3}$ Zellen $/ \mathrm{ml} \mathrm{S}$. epidermidis und $5 \times 10^{4}$ Zellen $/ \mathrm{ml}$ für $B$. subtilis konnte eine mehr als ausreichende Anzahl von Bakterien-Zellen auf der Chip-Oberfläche für die Raman-basierte Identifikation vorgefunden werden. Diese Werte wurden für eine Inkubationszeit von nur $10 \mathrm{~min}$ erhalten. Für diagnostische Zwecke ist eine höhere Empfindlichkeit wünschenswert. Dies könnte einerseits durch eine Erhöhung der Inkubationszeit und andererseits durch eine Vergrößerung des Probenvolumen (für die beschriebenen Versuche wurden $100 \mu \mathrm{l}$ eingesetzt) erreicht werden.

Tab. 1: Resultate der PCA/LDA

\begin{tabular}{|c|c|c|c|c|c|c|}
\hline Spezies & $\begin{array}{c}\text { K. } \\
\text { pneumoniae }\end{array}$ & $\begin{array}{c}P . \\
\text { aeruginosa }\end{array}$ & $\begin{array}{c}E . \\
\text { faecium }\end{array}$ & $\begin{array}{c}E . \\
\text { faecalis }\end{array}$ & $\begin{array}{c}\text { S. } \\
\text { aureus }\end{array}$ & $\begin{array}{c}\text { Spezifität } \\
{[\%]}\end{array}$ \\
\hline $\begin{array}{c}\text { K. } \\
\text { pneumoniae }\end{array}$ & $\mathbf{4 8}$ & 1 & 1 & 1 & 0 & 97 \\
\hline $\begin{array}{c}P . \\
\text { aeruginosa }\end{array}$ & 0 & 19 & 0 & 0 & 0 & 100 \\
\hline E. faecium & 0 & 0 & 26 & 5 & 0 & 96 \\
\hline E. faecalis & 0 & 1 & 2 & 18 & 1 & 97 \\
\hline $\begin{array}{c}\text { S. aureus } \\
\text { Sensitivität }\end{array}$ & 1 & 2 & 0 & 0 & 31 & 97 \\
\hline [\%] & 98 & 83 & 90 & 75 & 97 & $\begin{array}{c}\text { Genauigkeit } \\
90 \%\end{array}$ \\
\hline
\end{tabular}

Die erhaltene Nachweisgrenze ist vergleichbar mit anderen Ansätzen bei denen die ganze Zelle eingefangen wird. So werden beispielsweise für auf Oberflächenplasmonresonanz (SPR) basierenden Methoden Nachweisgrenzen zwischen $10^{5}$ bis $10^{7}$ Zellen/ml erreicht. [14,15] Ein generelles Hindernis für das Einfangen ganzer Bakterien in einem mikrofluidischen System stellen die fluidischen Kräfte dar, die es zu überwinden gilt. Dies bedeutet, die Bakterien müssen die ChipOberfläche erreichen und die Fängermoleküle sowie die Antikörper-Antigen-Bindung müssen der Scherkraft der laminaren Strömung standhalten. Da der Durchmesser der meisten bakteriellen Zellen in der Größenordnung von einigen Mikrometern liegt, sind diese Kräfte nicht unerheblich, und verringern die Empfindlichkeit der Assays. [16]

\section{Zusammenfassung}

In dieser proof-of-concept-Studie wurde ein neues, chipbasiertes Verfahren zur Isolierung und Anreicherung von Mikroorganismen vorgestellt. Unser Ansatz wird in Kombination mit der Raman-Mikroskopie zur Identifizierung 
von Bakterien verwendet. Es wurde gezeigt, dass mit nur zwei verschiedenen Antikörpern eine Vielzahl von verschiedenen Bakterienspezies auf dem Chip angereichert werden kann. Der entwickelte Chip ist vollständig kompatibel mit Ramanspektroskopischen Methoden. Dementsprechend konnten typische Spektren aller untersuchten Spezies erhalten werden. Ein Vergleich mit etablierten Nickelfolie-Substraten zeigte keine signifikanten Unterschiede in Bezug auf die erhaltenen Spektren. Weiterhin wurde gezeigt, dass die erfassten spektralen Daten für die Klassifizierung mittels PCA und LDA geeignet sind. In zukünftigen Experimenten wird die Anwendbarkeit des Verfahrens für komplexe Matrizen wie Blut, Urin oder andere Körperflüssigkeiten untersucht werden, welche für diagnostische Zwecke elementar sind. Im Erfolgsfall werden wir unsere Datenbank entsprechend erweitern, um so die schnelle und sichere Identifizierung einer Vielzahl von Bakterienarten zu ermöglichen.

\section{Danksagung}

Für die finanzielle Unterstützung der Forschungsprojekte 'FastDiagnosis' (13N11350) sowie der 'JBCI 2.0' (03IPT513Y Unternehmen Region, InnoProfile Transfer) danken wir dem Bundesministerium für Bildung und Forschung (BMBF), Deutschland. Für hilfreiche Diskussionen danken wir Carolin Steinbrücker.

[1] D. Threm, Y. Nazirizadeh, M. Gerken, Photonic crystal biosensors towards on-chip integration, Journal of Biophotonics 5, 601-616 (2012); doi: 10.1002/jbio.201200039

[2] P. Negri, R. A. Dluhy, Ag nanorod based surfaceenhanced Raman spectroscopy applied to bioanalytical sensing, Journal of Biophotonics 6 , 20-35 (2013); doi: 10.1002/jbio.201200133

[3] A. Kumar, D. Roberts, K. E. Wood, B. Light, J. E. Parrillo, S. Sharma, R. Suppes, D. Feinstein, S. Zanotti, L. Taiberg, D. Gurka, A. Kumar, M. Cheang, Duration of hypotension before initiation of effective antimicrobial therapy is the critical determinant of survival in human septic shock, Critical Care Medicine 34, 1589-1596 (2006); doi: 10.1097/01.CCM.0000217961.75225.E9

[4] R. P. Dellinger, M. M. Levy, J. M. Carlet, Surviving Sepsis Campaign: international guidelines for management of severe sepsis and septic shock: 2008, Critical Care Medicine 36, 1394-1396 (2008); doi: 10.1007/s00134-0122769-8

[5] S. Meisel, S. Stöckel, M. Elschner, F. Melzer, P. Rösch, J. Popp, Raman spectroscopy as potential tool to detect Brucella from milk, Applied and Environmental Microbiology 78, 5575-5583 (2012); doi: 10.1128/AEM.00637-12

[6] S. Pahlow, A. Maerz, B. Seise, K. Hartmann, I. Freitag, E. Kaemmer, R. Boehme, V. Deckert, K. Weber, D. Cialla, J. Popp, Bioanalytical application of surface- and tip-enhanced Raman spectroscopy, Engineering in Life Sciences 12, 131-143 (2012); doi: 10.1002/elsc.201100056

[7] M. Li, P. C. Ashok, K. Dholakia, W. E. Huang, Raman Activated Cell Counting for Profiling Carbon Dioxide Fixing Microorganisms, Journal of Physical Chemistry A 116, 6560-6563 (2012); doi: $10.1021 / \mathrm{jp} 212619 \mathrm{n}$

[8] J.-S. Maeng, N. Kim, C.-T. Kim, S. R. Han, Y. J. Lee, S.-W. Lee, M.-H. Lee, Y.-J. Cho, Rapid detection of food pathogens using RNA aptamers-immobilized slide, Journal of Nanoscience and Nanotechnology 12, 5138-5142 (2012); doi: 10.1166/jnn.2012.6369

[9] R Development Core Team, R Foundation for Statistical Computing, Vienna, Austria, 2011

[10] T. Bocklitz, A. Walter, K. Hartmann, P. Rösch, J. Popp, How to pre-process Raman spectra for reliable and stable models? Analytica Chimica Acta 704, 47-56 (2011); doi: 10.1016/j.aca.2011.06.043

[11] D. Zhang, K. N. Jallad, D. Ben-Amotz, Stripping of cosmic spike spectral artifacts using a new upper-bound spectrum algorithm, Applied Spectroscopy 55, 1523-1531 (2001); doi: 10.1366/0003702011953757

[12] T. Fearn, in Handbook of vibrational Spectroscopy, Vol. 3 (Eds.: J. M. Chalmers, P. R. Griffiths), John Wiley \& Sohn Ltd, Chichester, 2002, pp. 2086.

[13] A. Hengerer, E. Prohaska, J. Decker, S. Hauck, E. Yacoub, C. Kosslinger, U. Reischl, S. Drost, H. Wolf, in Trends and New Applications of Thin Films, Vol. 287-2, 1998, pp. 169.

[14] P. Skottrup, M. Nicolaisen, A. F. Justesen, Rapid Determination of Phytophthora infestans sporangia Using a Surface Plasmon Resonance Immunosensor, Journal of Microbiological Methods 68, 507-515 (2007); doi: 10.1016/j.mimet.2006.10.011

[15] S. Hearty, P. Leonard, J. Quinn, R. O'Kennedy, Production, characterisation and potential application of a novel monoclonal antibody for detection of virulent Listeria monocytogenes, Journal of Microbiological Methods 66, 294-312 (2006); doi: 10.1016/j.mimet.2005.12.009

[16] P. Leonard, S. Hearty, G. Wyatt, J. Quinn, R. O'Kennedy, Development of a surface plasmon resonance-based immunoassay for the direct detection of Listeria monocytogenes, Journal of Food Protection 68, 728-735 (2005); doi: 10.1016/j.bios.2003.11.009 